\title{
Slow Positron Beam Studies of the Stainless Steel Surface Exposed to Sandblasting
}

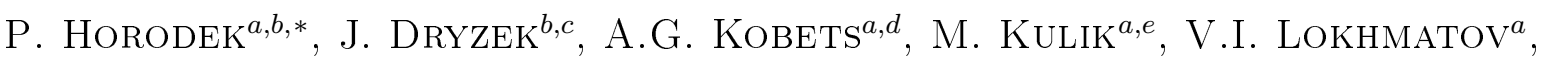

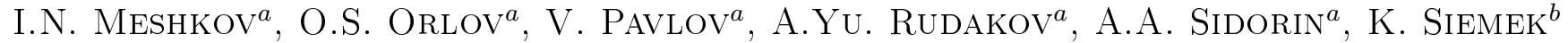 \\ AND S.L. YAKOVENKO ${ }^{a}$ \\ ${ }^{a}$ Joint Institute for Nuclear Research, Joliot-Curie 6, 141980 Dubna, Moscow region, Russia \\ ${ }^{b}$ Institute of Nuclear Physics, PAS, E. Radzikowskiego 152, 31-342 Kraków, Poland \\ ${ }^{c}$ Institute of Physics, Opole University, Oleska 48, 45-052 Opole, Poland \\ ${ }^{d}$ Institute of Electrophysics and Radiation Technologies, NAS of Ukraine \\ Chernyshevsky St. 28, 61002 Kharkov, Ukraine \\ ${ }^{e}$ Institute of Physics, Maria Curie-Skłodowska University, pl. M. Curie-Skłodowskiej 1, 20-031 Lublin, Poland
}

\begin{abstract}
The paper presents slow positron beam studies of the stainless steel grade 304 AISI samples annealed in the flow $\mathrm{N}_{2}$ atmosphere and sandblasted under different pressure from 1 to 7 bar. Heating of specimens caused formation of an additional layer on the surface which can be identified as oxides. Sandblasting reduces the thickness of the oxide layer and also defects concentration (vacancies as we suppose) decreases in dependence on pressure applied during blasting. Additionally, the atomic concentrations of oxygen have been obtained using nuclear methods (Rutherford backscattering and nuclear reactions) in the near surface layers of the studied samples.
\end{abstract}

DOI: $10.12693 /$ APhysPolA.125.714

PACS: 78.70.Bj, 82.80.Yc, 61.82.Bg

\section{Introduction}

Positron annihilation spectroscopy (PAS) is a sensitive method for detection of open volume defects such as vacancies, vacancy clusters and nanovoids [1]. It allows to recognize defects sized from 0.1 to $1 \mathrm{~nm}$ and their concentrations at the level of $10^{-7}$. For detection of point defects PAS seems to be more effective than X-ray diffraction (XRD) [2], scanning (SEM) or transmission electron microscopy (TEM) [3]. Nowadays, slow positron beams allow to study the adjoined surface layer of $c a .1 \mu \mathrm{m}$ thickness.

In this paper we point out that the slow positron beam applied to study samples of stainless steel grade 304 (AISI), which were annealed in a shield $\mathrm{N}_{2}$ atmosphere and sandblasted, allows to observe interesting changes on the surface. Stainless steel is a material often studied using PAS as well. The main aim of these investigations is to find defects appearing in the samples as a result of different physical processes. In most cases specimens are specially prepared to get the well described structure before its modification.

For example, Wu and Yan [4] annealed the samples of stainless steel 304 at $1073 \mathrm{~K}$ for 15 min under vacuum of $10^{-3} \mathrm{~Pa}$ and next deformed by cold-rolling and charged electrolytically with hydrogen. They showed that hydrogen damage between the surface and the bulk has a significant variation with depth and strongly depends on the condition of hydrogen-charging. The voids close to

*corresponding author; e-mail: Pawel.Horodek@ifj.edu.pl the surface and dislocations in the bulk after hydrogen-charging appeared.

Yabuuchi et al. [5] examined 304 AISI steel subjected to sensitization heat treatment, corrosion treatment and tensile plastic deformation. They noticed that the main source of defects such as vacancies is a plastic deformation. Additionally, they interpreted the observed defects as monovacancies, which were annealed at the temperature of $200-440^{\circ} \mathrm{C}$. However, to suppress chromium carbide participation, before deformations all the samples were annealed at $1150^{\circ} \mathrm{C}$ for $2 \mathrm{~h}$ in vacuum silica tube and subsequently quenched into ice water. It was the reference state for specimens.

Wang et al. [6] tested 316L steel subjected to surface mechanical attrition treatment. They observed changes in configuration of defects after cyclical removal of the layers near the surface. Their samples were not thermally prepared to get the initial bulk state because they focused on the changes after etching.

Dryzek et al. [7] in SW18 steel, using slow positron beam, determined diffusion lengths for the samples subjected to friction. In our previous research we established the effect of friction on the creation of surface layer in 304 steel [8]. Using measurements made on standard sources of ${ }^{22} \mathrm{Na}$ and ${ }^{68} \mathrm{Ge} /{ }^{68} \mathrm{Ga}$ we observed that the depth of a defected layer depends on the load applied during sliding and that the shape of profiles of defect concentrations differs from corresponding profiles for pure iron [9]. Measurements of the positron lifetimes showed the formation of vacancies close to or at dislocations. The thickness of worn layer extended from $85 \mu \mathrm{m}$ to $400 \mu \mathrm{m}$ depending on the applied load during tribo-tests. The influence of various industrial cutting techniques on the range of 
formation of defects was also compared [10]. Samples, in our previous studies $[8,10]$, were annealed to get the non-defected bulk state. However, any changes on the surface connected with annealing could not be observed using standard experiments.

It is a well-known situation that if a metal is heated until it glows, in air atmosphere it reacts with oxygen and unwelcome oxides cover can be formed [11]. To protect the steel from such phenomenon the application of the shield atmospheres (such as nitrogen, argon, or hydrogen) is recommended but they do not give the $100 \%$ protection and their usage depends on the annealed alloy.

The aim of this work is to examine with the use of slow positron beam the subsurface regions of the samples annealed in $\mathrm{N}_{2}$ and then sandblasted. Usually sandblasting, as the process of surface modification, is used to remove layers of oxides from the surfaces [12] or to create a desired surface texture. Diffusion of nitrogen atoms and then deformation induced by the sandblast treatment can generate a layer with defects. We are interested in the thickness of the layer and the defects concentration as well.

\section{Experimental details}

The object of the experiment were samples of stainless steel grade 304 AISI. The chemical composition is following: $0.06 \% \mathrm{C}, 1 \% \mathrm{Si}, 2.0 \% \mathrm{Mn}, 0.04 \% \mathrm{P}, 0.015 \% \mathrm{~S}$, $0.1 \% \mathrm{~N}, 17.0 \% \mathrm{Cr}$, and $10 \% \mathrm{Ni}$. At the beginning specimens in the shape of small discs of $5 \mathrm{~mm}$ thickness and $10 \mathrm{~mm}$ in diameter were mechanically polished to get the most even, clear, and shiny surface. Afterwards, they were annealed for $1 \mathrm{~h}$ at a temperature of $700^{\circ} \mathrm{C}$ in flow of $0.35 \mathrm{~cm}^{3} / \mathrm{s}$ atmosphere of $\mathrm{N}_{2}$. Subsequently all the samples were cooled to the room temperature in a furnace. As a result of heating, the surface of samples became similar to those presented by Naewkanya et al. [11], which suggests formation of oxides on the surface. The colourful layer was not removed. Such prepared samples were sandblasted using the Renfert Vario Basic Jet Blaster for $30 \mathrm{~s}$ under pressures of $1,3,5$, and 7 bar. We applied NANODENTAL abrasive particles with the size of $110 \mu \mathrm{m}$ in diameter. The mixture consisted of $\mathrm{Al}_{2} \mathrm{O}_{3}$ $(99.5 \%)$ and traces of $\mathrm{SiO}_{2}, \mathrm{Fe}_{2} \mathrm{O}_{3}, \mathrm{TiO}_{2}, \mathrm{CaO}, \mathrm{Na}_{2} \mathrm{O}$.

All samples have been tested on slow positron beam operating as a part of LEPTA project at JINR in Dubna [13]. The positrons are emitted from ${ }^{22} \mathrm{Na}$ source of $25 \mathrm{mCi}$ activity and moderated onto frozen $\mathrm{Ne}(7 \mathrm{~K})$ under pressure of $10^{-8}$ Torr. Moderated positrons are accelerated to the demanded energy in the range between $50 \mathrm{eV}$ and $35 \mathrm{keV}$. They are implanted into the sample in the shape of a beam of $3 \mathrm{~mm}$ diameter and intensity of $10^{5} \mathrm{e}^{+} / \mathrm{s}$. $511 \mathrm{keV}$ quanta from the annihilation process are registered by Doppler broadening of annihilation gamma line spectrometer (DBGL) with $1.2 \mathrm{keV}$ energy resolution interpolated to $511 \mathrm{keV}$.

From the measured lines the so-called $S$ parameter was extracted. It is defined as a ratio of area under the central part of the annihilation line to the total surface area under this line. Integration area is established once for all measurements. The $S$ parameter is usually near 0.5 . $S$ parameter is strictly correlated with the type and concentration of defects, i.e. the higher its value, the bigger concentration of defects. It is the basic parameter calculated in analysing of data obtained from DBGL measurements.

It is known that some elements in examined samples such as $\mathrm{Fe}, \mathrm{Cr}$, Ni combine with oxygen easily and form stable oxides. To prove it, after PAS measurements we used Rutherford backscattering spectrometry (RBS) and nuclear reactions ${ }^{16} \mathrm{O}(\alpha, \alpha){ }^{16} \mathrm{O}$ NR [14] to define concentration of oxygen in the near surface layer of the referential sample. The specimen was tested after annealing and after mechanical polishing of the surface. We polished the surface to remove visible changes on it created during heating. The RBS and ${ }^{16} \mathrm{O}(\alpha, \alpha){ }^{16} \mathrm{O}$ NR measurements were carried out using a $3045 \mathrm{keV} \mathrm{He}^{+}$ion beam and the scattering angle $170^{\circ}$.

\section{Results and discussion}

\subsection{PAS results}

Figure 1 shows the results of measurements using the variable energy positron beam for the referential sample (black circles) and samples sandblasted (white points) under different pressures. The calculated values of $S$ parameter are presented as a function of positron incident. Estimation of the mean range of positron penetration was calculated as follows:

$$
\bar{z}=\frac{A_{1 / 2}}{\rho} E^{n},
$$

where $\rho=7.8 \mathrm{~g} / \mathrm{cm}^{3}$ is the density of material, $A_{1 / 2}=$ $2.39 \mathrm{~nm} / \mathrm{keV}^{n}$ and $n=1.692$ are the Makhov parameters for $\mathrm{Fe}[15]$. Therefore, the minimal implantation depth for minimal energy used in experiment, it means $1 \mathrm{keV}$, is about $2.8 \mathrm{~nm}$ while the maximal depth equals approximately $1.3 \mu \mathrm{m}$ for $35 \mathrm{keV}$.

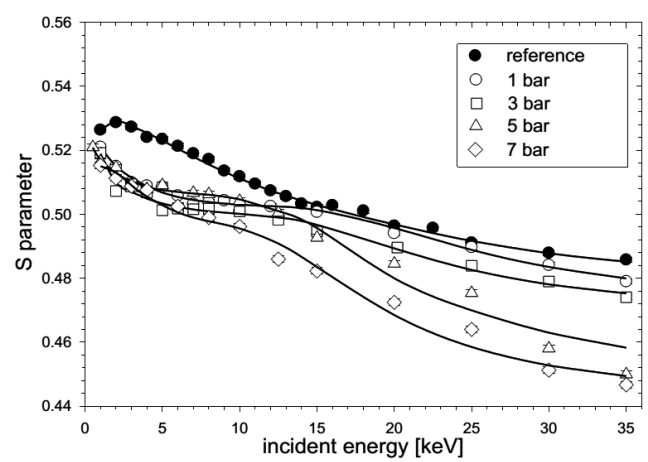

Fig. 1. The dependence of the measured $S$ parameter on the positron energy for the reference sample (black circles) and the sandblasted ones under pressure 1 bar (white circles), 3 bar (white squares), 5 bar (white triangles) and 7 bar (white diamonds). The solid lines represent the best fit of model curves obtained from VEPFIT [16].

The shape of the received profiles, presented in Fig. 1 is not typical and differs significantly from the ones available in literature [5-7], but for the referential sample 
(closed circles) there occurs the similarity between profile presented here and obtained by $\mathrm{Wu}$ et al. [17]. In their investigation the sample of pure iron was annealed at $1024 \mathrm{~K}$ for 15 min under a vacuum of $10^{-3} \mathrm{~Pa}$, next mechanically polished to remove oxide layers and deformed by cold-rolling. After that, the specimen was corroded in the electrolyte solution of $1 \mathrm{~N} \mathrm{H}_{2} \mathrm{SO}_{4}$ and destilled water under the anodic potential of $1.0 \mathrm{~V}$ for $10 \mathrm{~min}$ at room temperature. The measured $S$ parameters points for corroded sample had much more values in comparison to only rolled specimen.

In our studies the $S$ parameter profile for the referential sample lies under profiles of sandblasted ones. Additionally, we can observe ranges where decreasing $S$ parameter has different slopes. Between energies of $2 \mathrm{keV}$ and $15 \mathrm{keV}$ it decreases linearly. At $16 \mathrm{keV}$ energy there is another drop of a different kind, which seems to saturate at energies unavailable in this experiment. For the samples subjected to sandblasting (open symbols) layered character of profiles becomes more apparent. It indicates the existence of two layers of different properties. In the first layer they decrease and saturate at about $5 \mathrm{keV}$ energy, while in the further part, instead of maintaining $S$ parameter values on a constant level, another drop occurs, which marks the beginning of another layer. The beginning of occurrence of another layer varies and depends on the pressure used during sandblasting. The bigger the sandblasting pressure the thinner the first layer. It is also worth pointing out that all the profiles for the sandblasted samples are located below the profiles for the referential sample.

The description of the presented $S$ parameter profiles was performed with VEPFIT program [16], which is used to fit dependences of $S$ parameters from energy through numerical solving of the positron diffusion equation. The implementation of double-layer model allowing to apply the implantation profile based on the Makhov parameters for Fe [15], made it possible to fit model function to the profiles represented by solid black lines in Fig. 1. The main adjustable parameters are the thickness of the first layer and the diffusion length and $S_{\text {layer }}$ in both layers. The values of the parameters are gathered in Table.

\section{TABLE}

The values of the best fitted parameters in two layers obtained from the VEPFIT program [16] and calculated vacancies concentrations (in relative units: number of defects per number of atoms in the lattice) from Eq. (2).

\begin{tabular}{c|c|c|c|c|c|c|c}
\hline \hline \multirow{2}{*}{ Sample } & \multicolumn{4}{|c|}{ 1st layer } & \multicolumn{3}{c}{ 2nd layer } \\
\cline { 2 - 8 } & Thickness [nm] & $L_{+}[\mathrm{nm}]$ & $S_{\text {layer }}$ & $C_{\mathrm{v}}$ & $L_{+}[\mathrm{nm}]$ & $S_{\text {layer }}$ & $C_{\mathrm{v}}$ \\
\hline reference & $337(14)$ & $23(1)$ & $0.5081(7)$ & $3.16 \times 10^{-4}$ & $44 \pm 2$ & $0.4838(8)$ & $8.64 \times 10^{-5}$ \\
1 bar & $207(12)$ & $42(4)$ & $0.5110(5)$ & $9.48 \times 10^{-5}$ & $54 \pm 6$ & $0.4822(9)$ & $5.74 \times 10^{-5}$ \\
3 bar & $182(10)$ & $44(5)$ & $0.4986(4)$ & $8.64 \times 10^{-5}$ & $70 \pm 9$ & $0.4786(9)$ & $3.41 \times 10^{-5}$ \\
5 bar & $146(10)$ & $52(7)$ & $0.5050(5)$ & $6.18 \times 10^{-5}$ & $75 \pm 12$ & $0.4593(9)$ & $2.97 \times 10^{-5}$ \\
7 bar & $120(8)$ & $54(4)$ & $0.5048(5)$ & $5.73 \times 10^{-5}$ & $79 \pm 8$ & $0.4438(9)$ & $2.68 \times 10^{-5}$
\end{tabular}

Sandblasting causes the thickness of the first layer to diminish depending on the pressure applied during the process. The phenomenon can be explained by the fact that nitrogen atmosphere during annealing did not prevent formation of oxides or nitrides on the surface. Sandblasting, on the other hand, causes elimination of this layer. Lindell and Pettersson [12] studied different types of austenitic stainless steel annealed in production lines with the aim to characterize the oxides formation on the surface. They observed a thin multilayer of oxides lower than $1 \mu \mathrm{m}$ in the case of cold rolling and next annealing of the stainless steel. In the case of the hot rolled and reheated material the thickness of oxides was bigger than $1 \mu \mathrm{m}$. Additionally, they used shot blasting to remove the thickness of oxides.

Positron diffusion length presented in Table lengthens with the increase of pressure applied during sandblasting. In the work [18] Wu et al. present studies of samples of pure iron and SS 304 and 316 AISI electrocorroded under different potentials. They observed that changes of $S$ parameter depend on the potentials and time. Additionally, they point out that corroded SS as well as iron can produce vacancy-type defects. In the corroded iron both large-size defects (e.g voids) and small-size defects appear, while in SS only small-size defects occur. For these reasons we decided to calculate defects concentration only for vacancies. Using formula

$$
C=\left[\left(\frac{L_{\text {bulk }}}{L_{+}}\right)^{2}-1\right] /\left(\tau_{\text {bulk }} \mu\right),
$$

where $\tau_{\text {bulk }}=109.6 \mathrm{ps}$ [10], $\mu$ is the trapping coefficient for a single vacancy in pure Fe equal to $1.1 \times 10^{15} \mathrm{~s}^{-1}$ [19], $L_{\mathrm{bulk}}=142 \mathrm{~nm}$ [20] (the positron diffusion length in the bulk) the estimation is possible. The results of estimations for two layers are also presented in Table. Concentration of vacancies in both layers decreases with the increase of pressure applied during sandblasting. Sandblasting, therefore, seems to remove the surface layer that contains the defects created during annealing. It is an interesting result and needs more investigations especially for the deeper layer. On the other hand, the chemical etching for removing the first layer is also possible but it could introduce other changes and also the precise reduction would be very hard. 


\section{2. $R B S$ and $N R$ results}

The typical spectra of scattered $\mathrm{He}^{+}$ions collected for investigated samples are presented in Fig. 2. The black circles represent the specimen which was only annealed and covered with, as we suppose, the oxide layer. In the measurements presented above it was called the referential sample. The white circles come from the same sample but after removing of the layer created during heating. It was done using mechanical polishing. The solid line represents the best fit of obtained spectra. A border near the energy of about $1100 \mathrm{keV}$ was observed. It is presented in Fig. 2a. This bound corresponds to the scattered $\alpha$ particles on nucleus of oxygen atoms. That spectrum was measured for the referential sample (black circles). In Fig. $2 b$ the part of the spectra of RBS in the region near the energy $1100 \mathrm{keV}$ for both specimens is presented. It is found that the intensity of this line decreases in the collected spectrum measured for polished sample (white circles). The atomic concentration in the near surface layers was calculated using SIMNRA [21] and BS1 code [22]. It was determined that in the layer of $100 \mathrm{~nm}$ under surface the concentration of oxides in the annealed was about 40 times as much as in the layer of polished one.

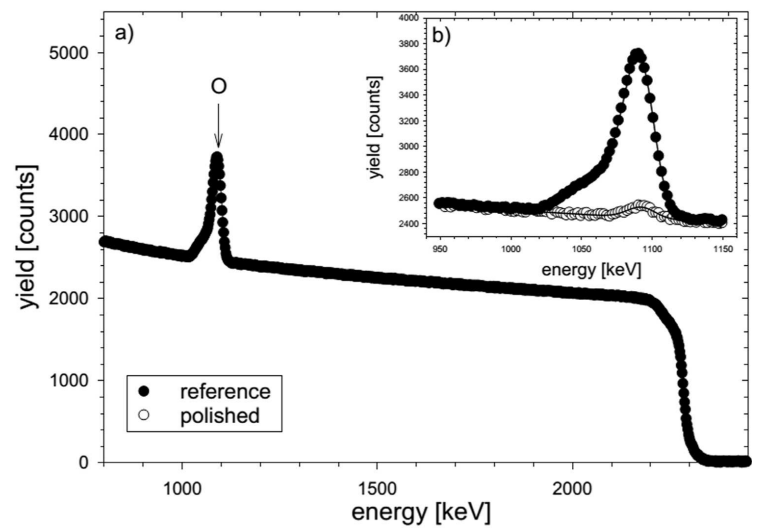

Fig. 2. RBS/NR spectra collected using a $3045 \mathrm{keV}$ $\mathrm{He}^{+}$ion beam and the scattering angle $170^{\circ}$ for sample after annealing called the reference sample (black circles) and the same sample after mechanical polishing (white circles). The solid line is the best fit of these spectra.

\section{Conclusions}

Nitrogen atmosphere during annealing seems to provide insufficient protection from formation of layers below the surface. The obtained profiles of $S$ parameter reveal the existence of at least two layers. Sandblasting diminishes the thickness of the first layer depending on pressure used during the process. Annealing in nitrogen atmosphere created multilayer of oxides on surface of samples which consisted of defects and sandblasting removed this layer exposing less defected areas.

\section{Acknowledgments}

Authors would like to thank Mrs Magdalena Maracewicz and Mr Marcin Maracewicz for the help with sample preparation.

All works were supported by RFBR grant No. 12-02-00072 .

\section{References}

[1] J. Kim, J. Byrne, J. Appl. Phys. 73, 2489 (2003).

[2] Y. Zhang, J. Liu, J. Wang, Y.J. Zeng, J. Strain Anal. 45, 319 (2010).

[3] M. De Vries, A. Mastenbroek, Met.Trans. A 8, 1497 (1977).

[4] Y.C. Wu, Y.C. Yan, Appl. Surf. Sci. 252, 3278 (2006).

[5] A. Yabuuchi, M. Maekawa, A. Kawasuso, J. Nucl. Mater. 419, 9 (2011).

[6] X. Wang, L. Xiong, X.G. Liu, G. Liu, J. Mater. Res. 25, 587 (2010).

[7] J. Dryzek, E. Dryzek, R. Krause-Rehberg, F. Reddmann, Tribol. Lett. 11, 121 (2001).

[8] J. Dryzek, P. Horodek, M. Wróbel, Wear 294-295, 264 (2012).

[9] J. Dryzek, Tribol. Lett. 42, 9 (2011).

[10] P. Horodek, J. Dryzek, M. Wróbel, Tribol. Lett. 45 341 (2012).

[11] P. Naewkanya, U. Panchareon, P. Labcharonwongsa, G. Lothongkum, J. Met. Mater. Miner. 20, 19 (2010).

[12] D. Lindell, R. Pettersson, Steel Research Int. 81, 542 (2010).

[13] E.V. Akhmanova, M.K. Eseev, A.G. Kobets, I.N. Meshkov, A.Yu. Rudakov, A.A. Sidorin, S.L. Yakovenko, Phys. Part. Nucl. Lett. 9, 373 (2012).

[14] Wei-Kan Chu, Backscattering Spectrometry, Academic Press, New York 1978.

[15] J. Dryzek, P. Horodek, Nucl. Instrum. Methods Phys. Res. B 266, 4000 (2008).

[16] A. Van Veen, A, H. Schut, M. Clement, A. Kruseman, M.R. Ijpma, J.M.M. De Nijs, Appl. Surf. Sci. 85, 216 (1995).

[17] Y.C. Wu, Y.Q. Chen, B. Wang, S.J. Wang, Y.C. Jean, R. Suzuki, T. Ohdaira, Appl. Surf. Sci. 252, 3274 (2006).

[18] Y.C. Wu, R. Zhang, H. Chen, Y. Li, J. Zhang, D.M. Zhu, Y.C. Jean, Radiat. Phys. Chem. 68, 599 (2003).

[19] A. Vehanen, P. Hautojarvi, J. Johansson, J. Yli-Kauppila, P. Moser, Phys. Rev. B 25, 762 (1982).

[20] F. Lukáč, J. Čižek, I. Procházka, Y. Jirásková, D. Janičkovič, W. Anwand, G. Brauer, J. Phys., Conf. Ser. 443, 012025 (2013).

[21] M. Mayer, SIMNRA User's Guide, http://simnra. sof tware. inf ormer.com/.

[22] V. Bohac, D.M. Shirokov, Nucl. Instrum. Methods Phys. Res. B 84, 497 (1994). 